\title{
LOS ESTILOS PARENTALES: IMPLICACIONES SOBRE EL RENDIMIENTO ESCOLAR EN ALUMNOS DE EDUCACIÓN MEDIA
}

\author{
María de la Luz Ortiz-Zavaleta y Omar Moreno-Almazán \\ UNAM, FES IZTACALA
}

MÉXICO

\begin{abstract}
RESUMEN
La investigación descriptiva y comparativa plantea como objetivo conocer las condiciones que derivan del estilo parental sobre el rendimiento académico de 90 adolescentes, ambos sexos, de segundo y tercer grado de una secundaria de San Juan del Río, Querétaro. El muestreo fue probabilístico aleatorio simple, con diseño no experimental, transversal-comparativo. Los instrumentos utilizados fueron el Cuestionario de Patrones de Autoridad Parental y la calificación promediada del primer bimestre. Los resultados del ANOVA indican que en $37.7 \%$ de las familias los padres ejercen el mismo estilo parental: $32.3 \%$ autoritarios con hijos con mejor rendimiento académico, seguido por $52.9 \%$ de padres democráticos, y $14.7 \%$ de padres negligentes con hijos con rendimiento deficiente. En $62.3 \%$ de las familias ambos padres educan con distinto estilo, y el rendimiento de los hijos no difiere significativamente. Conclusión: quienes sean educados por padres de mismo estilo parental tendrán una línea marcada en su rendimiento académico en función a dicho estilo.
\end{abstract}

Palabras Clave:

Adolescencia, educación media, estilos parentales, rendimiento académico, evaluación psicológica.

\section{PARENTAL STYLES: IMPLICATIONS ON SCHOOL PERFORMANCE IN MIDDLE EDUCATION STUDENTS}

\begin{abstract}
This descriptive and comparative research aims understand the conditions that derive from parenting style on academic performance of 90 adolescents, both sexes, second and third secondary of San Juan del Río, Querétaro. Probabilistic sampling was simple random, non-experimental design with cross - comparative. The instruments used were the Questionnaire Patterns of Parental Authority and the averaged score the first two months. ANOVA results indicate that $37.7 \%$ of families parents exercise parental same style: authoritarian $32.3 \%$ of children with better academic performance, followed by $52.9 \%$ of parents democratic; and $14.7 \%$ neglectful parents of children with poor performance. In $62.3 \%$ of families with both parents educate different style and performance of the children does not differ significantly. Conclusion: who are educated by parents of the same parenting style will have a line marked on their academic performance according to that style.
\end{abstract}

Keywords:

Adolescence, middle education, parentings style, academic performance, psychological evaluation

Bitácora del Artículo:

| Recibido: 3 de Agosto de 2015 | Aceptado: 3 de Septiembre de 2015 | Publicado en línea: Enero-Junio de 2016 | 


\title{
ESTILOS PARENTALES: IMPLICACIONES SOBRE EL RENDIMIENTO ESCOLAR
}

\author{
María de la Luz Ortiz-Zavaleta y Omar Moreno-Almazán \\ UNAM \\ MÉXICO
}

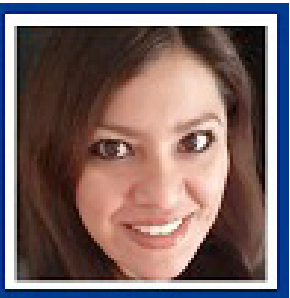

María de la Luz Ortiz Zabaleta

FESI, UNAM

Correo: mluz.oz@hotmail.com

Licenciada en Psicología, FESI, UNAM. Actualmente se desempeña como psicóloga clínica.

Ver más...

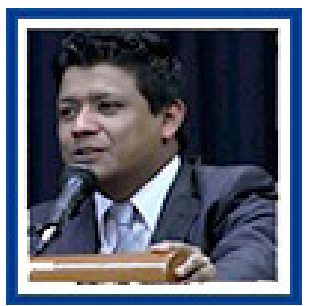

Omar Moreno Almazán

FESI, UNAM

Correo: almazanomar@gmail.com

Doctor en Psicología en el área de conocimiento de Psicología Educativa y del Desarrollo en la UNAM. Actualmente se desempeña como docente universitario, psicoterapeuta y perito en materia civil y penal.

Ver más...

\section{CONTRIBUCión DE lOS AutORES}

La primera autora, María de la Luz Ortiz Zabaleta elaboró el fundamento teórico, realizó la aplicación de los instrumentos y elaboró el análisis de resultados.| Omar Moreno Almazan participó en la función de supervisión y revisión del documento.

\section{AGRADECIMIENTOS}

Los autores agradecen a la Escuela Secundaria General "Antonio Caso", de San Juan del Río, Querétaro, por su colaboración en la elaboración del presente trabajo.

\section{Datos de Filiación de los Autores}

FES Iztacala, UNAM| FES Iztacala, UNAM

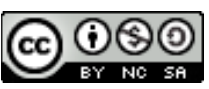

Copyright: (c) 2016 Ortiz-Zabaleta, M.L. \& Moreno-Amazán,

Este es un artículo de acceso abierto distribuido bajo los términos de la licencia Creative Commons Reconocimiento-NoComercial -Compartirlgual 4.0 Internacional, por lo que su contenido gráfico y escrito se puede compartir, copiar y redistribuir total o parcialmente sin necesidad de permiso expreso de sus autoras con la única condición de que no se puede usar con fines directamente comerciales y los términos legales de cualquier trabajo derivado deben ser los mismos que se expresan en la presente declaración. La única condición es que se cite la fuente con referencia a la Revista Digital Internacional de Psicología y Ciencia Social y a sus autoras. 


\section{TABLA DE CONTENIDO}

tipo y diseño de investigación, 82

Participantes, 82

Selección de la muestra, 82

Definición de variables, 82

1. Variable independiente: estilos parentales, 82

2. Variable dependiente: rendimiento escolar, 82

Hipótesis, 83

Instrumentos, 83

1. Cuestionario de Patrones de Autoridad Parental (CPAP) de Aguilar, Valencia y Romero (2007)., 83

Procedimiento, 83

RESULTADOS

Rendimiento, 83

Condición familiar y estilos parentales percibidos, 84

DisCUSIÓN Y CONCLUSIONES 


\section{INTRODUCCIÓN}

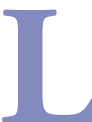

a educación es fundamental para la transmisión de la cultura en que se desarrolla el ser humano, además de que prepara al individuo para la convivencia social (Díaz y Morales, 2011). Por tanto, la educación escolarizada es una pieza fundamental, y aunque muchas veces es vista como proveedora de información pasiva, su alcance es mayor, pues proporciona herramientas para el desarrollo y enriquecimiento de la personalidad, habilidades, destrezas, aptitudes, ideales, intereses y valores (SEP, 2012), lo cual es necesario para el desarrollo de sociedades sanas.

El método que utilizan las instituciones educativas para determinar si un alumno está aprendiendo lo que necesita para su desarrollo es la evaluación educativa, la cual mide - a través de una prueba- el conocimiento del estudiante y lo compara con un parámetro que establece lo que a su edad debería saber; a este resultado se le denomina "rendimiento escolar" (INEE, 2003).

Un problema evidente en México se muestran en los porcentajes nacionales de alumnos de educación básica en el rubro de rendimiento insuficiente y elemental, el cual es preocupante pues los indicadores señalan que para 2013 en la materia matemáticas se tenía $51.2 \%$ de alumnos de primaria en este rubro, y $78.1 \%$ de alumnos en el nivel secundaria; en el área de español se obtuvo $57.2 \%$ de alumnos de primaria y $80.3 \%$ de alumnos de secundaria con insuficiente y elemental rendimiento; esto muestra los peores resultados en el nivel secundaria (SEP, 2012).

Estos indicadores han generado gran interés en el fenómeno del bajo rendimiento escolar, el cual es definido por Enríquez, Segura y Tovar (2013) como la imposibilidad del alumno por alcanzar un resultado favorable en la evaluación educativa, lo que indica que su preparación no es la suficiente para que logre vivir de forma autónoma y al mismo tiempo comportarse de manera cívica, responsable y tolerante en la sociedad.

Sumado a la preocupación por el fenómeno de bajo rendimiento, se evidencia que la etiología es bastante compleja, pues hay una gran variedad de factores que intervienen y que generalmente actúan de manera conjunta (Enríquez, Segura y Tovar, 2013). Los factores que se han encontrado involucrados son fisiológicos, pedagógicos, psicológicos y sociales. Los fisiológicos se refieren a problemas endocrinológicos, déficit en órganos de los sentidos (auditivo, visual, motor, lenguaje, etc.) (Guall- pa y Loja, 2015); también se incluyen en este rubro las enfermedades físicas y la desnutrición (Enríquez, Segura y Tovar, 2013). Los factores pedagógicos refieren al desempeño de los profesores y del propio sistema educativo (Guallpa y Loja, 2015), pero también se incluyen las condiciones de aula y los servicios de apoyo (asesorías, orientación educativa y becas) (Becerra, 2012). Los factores psicológicos se refieren a las características cognitivas, afectivas y conductuales que originan trastornos que obstaculizan el aprendizaje (Guallpa y Loja, 2015), además de las estrategias de afrontamiento, aptitudes, inteligencia y motivación de logro (Becerra, 2012). Los factores sociales tienen una gama mucho más amplia, pues abarca el entorno familiar, cultura, medios de comunicación, etc.; dentro del entorno familiar se derivan el nivel socioeconómico, estilos parentales, estructura familiar, etc. (Enríquez, Segura y Tovar, 2013; Linares, 2010).

Así, un elemento sumamente importante para el estudio es la familia, pues ésta es la primera influencia que tiene el individuo desde su nacimiento y la proveedora de las principales herramientas que enriquecen su personalidad (González, 2008; Bermejo, 2011; CONAFE, 2012), la cual es la base fundamental que determina el comportamiento de los individuos.

Se apunta a que en el aprendizaje el individuo también es responsable de poseer el deseo de aprender y entender, de esforzarse y ser perseverante; todos estos conceptos son características de lo que se denomina orientación a la meta, pero el desarrollo de estas condiciones suele generar complicación dentro del mismo entorno familiar; de este modo se entiende que una de las variables con mayor influencia en el rendimiento académico son los estilos parentales, pues se ha demostrado que los niños con maltrato infantil presentan una tendencia de 2.5 veces mayor para repetir el año escolar (Enríquez, Segura y Tovar, 2013).

El papel de los padres sobre el desempeño escolar parece ser determinante respecto a múltiples elementos que van más allá del tipo de estructura familiar que tengan, en virtud de que su actuación estará ofreciendo a sus hijos un repertorio conductual mucho más amplio y contribuyendo a que éstos se vean favorecidos en su desarrollo; esta es la verdadera importancia de que ambos padres trabajen en conjunto, poniéndose de común acuerdo para elegir la forma más adecuada para criarlos (Díaz y Morales, 2011).

En el aspecto escolar, la motivación trae consigo un aumento en la participación del alumno en clase, el disfrute e interés por las tareas, además de un alto nivel de creatividad y flexibilidad cognitiva; es así que este concepto va de la mano con la autonomía, pues cuando el 
padre promueve la autonomía del hijo, éste tendrá una mayor motivación, competencia y persistencia (Kleisner y MacPhee, 2011). Pero, como menciona Edel (2003), la motivación necesita un factor externo que fomente los recursos de competencia, y las personas más idóneas para esta contribución son los padres, evidenciando de nuevo la importancia de la familia en el desarrollo de buenas competencias escolares.

Otro concepto importante que se desarrolla en el seno familiar es el autoconcepto, el cual se define como el sistema de creencias que un individuo considera verdaderas respecto a sí mismo, y que está determinado por el análisis que hace de su persona en conjunto con lo que los demás piensan de él (Rodríguez y Caño, 2012). Por tanto, el autoconcepto académico es la evaluación que hace el sujeto de su propia capacidad para aprender en el contexto escolar (Ishak, Low y Lau, 2011). En la infancia temprana es cuando dichos conceptos se desarrollan, habiendo influencia de los estilos de los padres, que pueden desde promoverlo hasta afectarlo, reflejándose en el rendimiento académico (Duchesne y Ratelle, 2010).

En este sentido, es posible saber que la autonomía de los alumnos deriva de la influencia paterna, haciéndose evidente en aspectos como la lectura y matemáticas; debido a que los chicos tienen mayor desarrollo en la responsabilidad social, reflexionan más acerca de su propia conducta, tienen interacciones positivas con los compañeros, tienen mayor motivación para aprender y capacidad para trabajar independientemente (Kleisner y MacPhee, 2011).

A su vez, otro concepto fundamental es la autoestima, definida como la percepción que se tiene de sí mismo, pero encaminado hacia el amor propio y la autoapreciación; involucra el respeto y el valor que uno mismo se da, además de la confianza de que nuestros actos son útiles en la vida; incide de forma importante en los individuos dado que les permite tener más ambición por el alcance de las metas escolares y de reconocer los sentimientos y pulsiones tanto positivas y negativas, lo cual lleva a tomar decisiones maduras (Madrigales, 2012).

Algunos estudios evidencian que los alumnos que tienen alto rendimiento escolar provienen de hogares con estilo autoritativo y se caracterizan por tener elevada madurez y autocontrol (Raya, 2008); sin embargo, también se ha señalado que para lograr un buen rendimiento escolar es necesario un óptimo autocontrol interno, pues éste ayuda a comprender que los éxitos y fracasos son productos de la inteligencia y esfuerzo; el bajo autocontrol encamina al estudiante a creer que el éxito y el fracaso son debido a la suerte, lo que disminuye su participación en sus tareas escolares y, por ende, la afectación en el rendimiento de éstas (Edel, 2003). A su vez, el autocontrol es necesario para la modulación de las conductas perjudiciales a la hora de estudiar, y tiene un nexo muy estrecho con la disciplina; ambos ayudan al estudiante para que adopte conductas razonadas que le Ilevarán a poner atención, seguir reglas, ser responsable en sus deberes y encaminarse de nuevo cuando algunas situaciones atenten contra sus objetivos (Raya, 2008).

Es necesario que padres de familia entiendan qué son los estilos parentales, pero para comprenderlos es necesario definir la práctica de crianza, que no es una más que una conducta o acción de la que se valen los padres para tener una interacción con sus hijos, las cuales son gobernadas por las dimensiones afecto y control (Duchesne y Ratelle, 2010; Clerici y García, 2011); por tanto, los estilos parentales son el conjunto o constelación de dichas conductas y actitudes del adulto, que al ser trasmitidas a los hijos les crea un clima emocional que determinará el desarrollo de su comportamiento y el tipo de vínculo que tendrá con sus padres (Ishak, Low y Lau, 2011; Raya, 2008; Climent, 2009).

El auge del estudio de los estilos parentales se dio en la década de los sesenta, cuando Diana Baumrid destacó la influencia que se hacía en el desarrollo de los hijos, y a partir de esto se establecieron los primeros tres modelos: autoritativo o democrático, autoritario y permisivo. Más tarde, en la década de los ochenta, Eleanor Maccoby y John Martin observaron que el estilo permisivo presentaba dos formas diferentes: indulgente y negligente; de esta forma surgió el cuarto estilo, el cual se denominó "negligente" (Raya, 2008; Torio, Peña y Rodríguez, 2008; Clerici y García, 2011).

Así, en el estilo democrático los padres tienen alto grado de exigencia, pero siempre explicando razones, establecen reglas y son firmes en ellas, hacen uso de sanciones adecuadas, pero están dispuestos a escuchar a sus hijos y llegar a acuerdos, apoyan la individualidad, los ayudan a ser independientes y les ofrecen orientación (Ramírez, 2005). Asimismo, promueven la comunicación familiar y respetan los derechos de sus hijos, pero también los propios; son sensibles a las peticiones y sentimientos de sus hijos. Uno de los aspectos más importantes es que estos padres se ajustan a las demandas de sus hijos de acuerdo con los niveles de desarrollo de éstos (Climent, 2009). Como consecuencia, se forman individuos con óptimas competencias sociales, con índices más altos en la autoestima, autoconcepto y bienestar psicológico, además de un menor nivel de conflictos con sus padres; suelen ser chicos interactivos, cariñosos, confiados, asertivos e independientes; todo esto contribuye a que en el ámbito escolar logren mejores rendimientos académicos (Torio, Peña y Rodríguez, 2008). 
En el estilo autoritario los padres se caracterizan por ser muy exigentes y valoran la obediencia estricta, pero prestan muy poca atención a las necesidades de sus hijos; imponen reglas y órdenes en demasía, las cuales no pueden ser cuestionadas ni negociadas (Climent, 2009); con frecuencia usan el castigo, entre ellos el físico (Raya, 2008; Ramírez, 2005). No fomentan la comunicación bidireccional, y cuando hablan es para enfatizar que ellos son la autoridad, por lo que nunca estimulan la independencia e individualidad, no dan afecto explícito y dedican gran parte del tiempo a influir, evaluar y controlar el comportamiento y actitudes de los hijos (Climent, 2009). Las consecuencias son hijos con bajas competencias sociales, tienen baja autonomía, son temerosos, irritables pero obedientes, con baja autoestima, reservados, poco comunicativos, poco afectuosos y tienden a una pobre interiorización de valores morales; respecto a las cuestiones escolares, son alumnos poco tenaces a la hora de perseguir metas y no hacen un análisis razonado; tienen poca creatividad, curiosidad y originalidad; pero se ha encontrado que no son los peores alumnos en cuanto a rendimiento escolar, pues su régimen tan estricto les obliga a dar buenos resultados (Torio, Peña y Rodríguez, 2008).

Los padres permisivos tienen poco control a la hora de guiar a sus hijos y exigen muy poca madurez, evitan lo más posible la afirmación de autoridad y la imposición de restricciones, y no son capaces de establecer normas, tareas u horarios (Raya, 2008). Hacen muy poco uso de castigos y toleran en demasía los impulsos de sus hijos, accediendo fácilmente a sus deseos; sin embargo, tienen comunicación abierta, atienden las necesidades de los hijos, son afectuosos, alientan la individualidad e independencia; todo esto crea hijos con altos índices de espontaneidad, originalidad, creatividad y autoestima, pero se reportan índices bajos en logros escolares debido a que tienen muy baja autorresponsabilidad, autocontrol y autoconfianza; también tienen mayor probabilidad de desarrollar conductas agresivas, poca independencia personal y poca madurez (Torio, Peña y Rodríguez, 2008).

El estilo negligente lo conforman los padres con pobre o nula implicación afectiva hacia sus hijos y un alto nivel de rechazo y hostilidad; no son exigentes y no ponen normas; por el contrario, son muy permisivos, pero por falta de tiempo, poco interés y por negligencia o comodidad. Educan de la manera más rápida y cómoda, no establecen diálogo y vigilancia, y tienden a ofrecer cosas materiales para halagar a sus hijos. Este estilo causa en los hijos los efectos más negativos en la socialización, desarrollan muy baja autoestima y autoconcepto, son bastante agresivos y muestran poco autocontrol, así como el peor desarrollo cognitivo, y por tanto bajos logros escolares; son niños que carecen de autonomía positiva, ya que hacen uso de su libertad irresponsablemente (Torio, Peña y Rodríguez, 2008).

Investigaciones como la del PISA (2011) identificaron en 2009 que los alumnos con mayor rendimiento en lectura son aquellos cuyos padres les leyeron a menudo durante el primer año de su educación primaria. También se encontró que los padres no necesitan tener altos grados académicos ni dedicar infinidad de horas para hacer las tareas con los hijos, lo que demuestra que el involucramiento paterno es muy importante para el desarrollo cognitivo. Esto último fue respaldado cuando se encontró que los adolescentes con mejor promedio académico eran aquellos con quienes sus padres hablaban con ellos acerca de cómo les había ido en su día, a los que les contaban historias, etc.; también se destacó la importancia de esta interacción aún en la adolescencia, pues los alumnos con más ventajas son aquellos que platican con sus padres sobre temas de política, temas sociales y de economía, pero también de temas informales (como de películas y programas de televisión); es decir, simplemente tener comunicación e interés.

Con lo anterior, y de manera empírica, parece que el modelo más adecuado es el democrático, pues beneficia a los niños y jóvenes no sólo en el aspecto cognitivo, sino en todo su desarrollo; es así que los padres necesitan aprender a educar con la misma proporción de amor y disciplina, pues esto les ayudará a ser adultos seguros, serenos y con capacidad de reflexionar; es necesario que comprendan que los hijos simplemente necesitan ser direccionados hacia el amor, límites, aceptación, respeto y demás valores que le ayudarán a ser mejores ciudadanos (Torio, Peña y Rodríguez, 2008).

Diversas líneas procuran brindar elementos que sirvan como recomendaciones para el ejercicio de los padres para que modifiquen sus estilos parentales, reduciendo el uso de castigos ante las conductas inadecuadas de sus hijos e incrementando el control inductivo, pues esta es la mejor forma en que pueden transmitir sus expectativas a los hijos sin que éstos lo perciban agresivo; también le permitirá entender por qué las reglas son necesarias, por qué una mala conducta es inaceptable, cómo su conducta afecta a los demás, qué debe hacer para que su conducta sea aceptable y cómo enmendar cualquier daño que pudieran haber ocasionado (Roksa y Potter, 2011).

Con base en los argumentos presentados, nos queda constancia de que la dinámica que se efectúa de los estilos parentales tienen una posible influencia en 
el rendimiento escolar de los adolescentes, lo cual, de ser cierto, proporciona un elemento notable que permite ampliar las estrategias de intervención dentro del campo clínico y educativo, dentro del margen de acción propio a nuestro contexto. Por esta razón, el presente estudio tuvo como objetivo conocer la presencia de los distintos perfiles parentales a partir de los estilos de crianza generados, y posteriormente analizar las diferencias que existen respecto al rendimiento escolar, en función de cada subgrupo de estilos parentales percibidos.

\section{MÉtodo}

\section{Tipo y diseño de investigación}

Es una investigación basada en un enfoque cuantitativo, de tipo descriptiva y comparativa. De acuerdo con Hernández, Fernández y Baptista (1991), las investigaciones descriptivas miden de manera independiente las variables involucradas, y por medio de una integración se describe cómo se manifiesta el fenómeno; tiene un diseño no experimental, transeccional o transversal, ex post facto.

\section{Participantes}

La población de estudio estuvo constituida por los alumnos de segundo y tercer grados del turno matutino de la secundaria General Antonio Caso, ubicado en la ciudad de San Juan del Río, Querétaro.

Se trabajó con alumnos de segundo y tercer grados considerando que ellos han adquirido mayor adaptación al sistema de trabajo de su institución; no se incluyó a alumnos de primer grado en virtud de que ellos aún comenzarán la adaptación al sistema de estudio de la institución. De esta forma, la muestra fue de $\mathrm{N}=90$ adolescentes, de los cuales 37 fueron hombres y 53 mujeres, con un rango de edad de 13 a 15 años.

\section{Selección de la muestra}

La selección de la muestra se realizó por medio del muestreo probabilístico aleatorio simple, una vez aplicados los criterios de exclusión.

\section{Pasos:}

1. Se imprimieron las listas de asistencia de los alumnos de segundo y tercer grados.

2. Se recortaron los nombres y se introdujeron en una urna para seleccionar 90 alumnos.

3. Se elaboró una lista con los nombres de los participantes, el grado y el grupo a que pertenecían.

\section{Definición de variables}

\section{Variable independiente: estilos parentales}

\section{Definición conceptual}

Son una constelación de actitudes de los padres que involucra las dimensiones de apoyo (amor, aprobación, ayuda) y control parental (disciplina, supervisión, normas) que son comunicadas a los hijos y que, en conjunto, crean un clima emocional que determinarán los comportamientos del hijo y los vínculos de éstos con sus padres (Climent, 2009).

\section{Definición operacional}

Los estilos parentales pueden medirse desde dos percepciones: 1) desde la opinión de los mismos padres, y 2) a través de la percepción de los hijos. Para este caso se utilizó la percepción de los hijos, utilizando el Cuestionario de Patrones de Autoridad Parental de Aguilar, Valencia y Romero (2007). Se tomó la definición de estilos a partir de lo observado por el instrumento, donde se hace una clasificación a partir de las categorías: democrático, autoritario, permisivo o negligente. Para estos fines se entiende que el padre democrático es el que adopta con sus hijos prácticas de crianza que se caracterizan por alto nivel de afecto y control; el padre autoritario se caracteriza por emitir prácticas de crianza con mayor nivel de control y bajo en afecto; el padre permisivo efectúa sus prácticas de crianza con alto grado de afecto, pero bajo en control; $y$, el padre negligente es quien en sus prácticas de crianza muestra bajo nivel de control y afecto.

\section{Variable dependiente: rendimiento escolar}

\section{Definición conceptual}

Se entiende como el producto de la comparación del resultado que arroja la medición del conocimiento del estudiante - el cual se realiza a través de la aplicación de una prueba - con el parámetro que establece lo que cada alumno debería saber (INEE, 2003). Para el caso de la educación media, los parámetros se basan en la adquisición de conocimientos esperados, los cuales son establecidos en los planes de estudio vigentes.

\section{Definición operacional}

El rendimiento escolar puede ser medido a través de las calificaciones escolares promediadas que asignan los profesores o las instituciones escolares (INEE, 2003). Éstas permiten comparar si el alumno tiene un rendimien- 
to "deficiente" = 5 y 6 , "regular" = 7, "bueno" $=8$, o "excelente" = 9 y 10 .

\section{Hipótesis}

- H1: Existirán diferencias significativas en el rendimiento escolar de los alumnos que tengan padres o cuidadores con el mismo estilo parental en comparación con aquellos cuyos padres o cuidadores difieran en estilo parental.

- Ho: No existirán diferencias significativas en el rendimiento escolar de los alumnos que tengan padres o cuidadores con el mismo estilo parental en comparación con aquellos cuyos padres o cuidadores difieran en estilo parental

- H2: Existirán diferencias significativas en el rendimiento escolar de alumnos que perciban a sus padres o cuidadores como democráticos en comparación de aquellos alumnos que perciban a sus padres con estilo autoritario, permisivo y negligente.

- H0: No existirán diferencias significativas en el rendimiento escolar de alumnos que perciban a sus padres o cuidadores como democráticos en comparación de aquellos alumnos que perciban a sus padres con estilo autoritario, permisivo y negligente.

\section{Instrumentos}

1. Cuestionario de Patrones de Autoridad Parental (CPAP) de Aguilar, Valencia y Romero (2007).

Se trata de un cuestionario aplicable a adolescentes, el cual evalúa las actitudes y prácticas parentales. Un total de 22 ítems evalúa si el padre tiene estilo de crianza democrático (5 ítem, $\mathrm{a}=.70$ ), autoritario (6 ítem, $a=.72$ ), permisivo (4 ítems, $a=.66$ ) o negligente (7 ítems, $a=.88$ ). Para calificar a la madre se tiene un total de 23 ítems que evalúan si ésta tiene estilo parental democrático (6 ítems, $a=.83$ ), autoritario ( 6 ítems, $a=$ .83 ), permisivo (3 ítems, $a=.60$ ) o negligente (8 ítems, $a=.92)$. La escala tipo Likert tiene como respuestas $4=$ totalmente cierto, $3=$ medianamente cierto, $2=$ poco cierto y $1=$ muy poco cierto, en donde a mayor puntaje mayor percepción del estilo parental. La consistencia interna global del cuestionario para el padre es de .81 alfa de Cronbach; y para el cuestionario de la madre es de .71 alfa de Cronbach.

2. Para medir el rendimiento académico se utilizó la calificación promediada bimestral del presente ciclo escolar.

\section{Procedimiento}

Los instrumentos se aplicaron de forma colectiva durante horarios de clase, contando con el consentimiento informado de las autoridades de la escuela y de los participantes. Los resultados se analizaron con apoyo del paquete estadístico SPSS y se trataron bajo análisis descriptivo y análisis de varianza con prueba post hoc.

\section{Resultados}

Para dar cumplimiento a los objetivos, se ha dividido esta sección en dos partes: 1) se expone la descripción de los constructos evaluados mediante los procesos estadísticos descriptivos, y 2) se hace un análisis comparativo entre los estilos parentales en la población.

\section{Rendimiento}

Para el caso de esta muestra, se tiene un rendimiento promedio de 7.4 de acuerdo con la escala establecida por la SEP para evaluarlo; teniendo calificaciones que van desde un promedio de 5 y hasta un máximo de 9 , con una desviación estándar de 1.079, la cual indica poca variación de datos. Los puntajes de mayor frecuencia son los que se sitúan dentro del rango de calificación de entre 5 y hasta 7 en su último periodo evaluado.

Las materias que resultan más difíciles para los estudiantes son español (con $27.8 \%$ ) y matemáticas (con $32.2 \%$ ); las dos anteriores con $7.8 \%, 2.2 \%$ dice que ninguna, y $30 \%$ dice que otras, dentro de las cuales figuran inglés, geografía, historia, biología y física. Una de las razones más frecuentes acerca de la dificultad es porque señalan que no tienen el conocimiento para hacer las actividades con $40.4 \%$, mientras que $2.2 \%$ señala que es porque no tiene apoyo de sus padres cuando tienen dudas; $13.5 \%$ contestó que porque le da flojera hacer las actividades de la materia, $12.4 \%$ porque no pone atención en clases, $10.1 \%$ indica que es debido a que el maestro no explica lo suficiente, $1.1 \%$ porque no le interesa estudiar, y $18 \%$ debido a otras causas (poca participación, enfermedad, no hay adaptación con la escuela y exceso de actividades escolares).

\section{Condición familiar y estilos parentales percibidos}

De los jóvenes de esta muestra, $72.3 \%$ vive con ambos padres, $23.3 \%$ sólo con la madre, 3.3\% sólo con el padre, y $1 \%$ con un familiar distinto. En cuanto a la supervisión escolar del padre, la frecuencia de 55 se aglomera en los rubros de "nunca" y "a veces"; por otro lado, con una frecuencia de 59 indica que las madres supervisan "casi siempre" y "siempre". 
En la tabla 1 se muestra la frecuencia de las respuestas de los adolescentes respecto a con quién viven y el estilo parental que perciben. El $37.8 \%$ percibe a ambos padres con igual estilo de crianza, $43.3 \%$ percibe que sus padres tienen distinto estilo, $15.5 \%$ tiene la influencia únicamente del estilo de su madre, $2.2 \%$ sólo tiene la influencia del estilo de su padre y $1.1 \%$ de un familiar distinto.

Observando sólo la frecuencia de alumnos que perciben a ambos padres con el mismo estilo parental, la tabla 1 indica que 18 alumnos (52.9\%) los percibieron como democráticos, 11 alumnos (32.3\%) como autoritarios, y 5 alumnos (14.7\%) como negligentes. El estilo permisivo no figuró en esta investigación. Finalmente, de los alumnos que tienen únicamente la influencia del estilo de su madre, se reporta que $21.4 \%$ la percibe como negligente, $7.6 \%$ como autoritaria y $71 \%$ como democrática.

Tabla 1.

Frecuencia de percepción de estilos parentales.

\begin{tabular}{|c|c|c|c|c|}
\hline EstiLos & $\begin{array}{l}\text { VIVEN } \\
\text { CON } \\
\text { AMBOS } \\
\text { PADRES }\end{array}$ & $\begin{array}{l}\text { VIVEN } \\
\text { SÓLO CON } \\
\text { MAMÁ }\end{array}$ & $\begin{array}{l}\text { VIVEN } \\
\text { SÓLO } \\
\text { CON } \\
\text { PAPÁ }\end{array}$ & $\begin{array}{l}\text { VIVEN } \\
\text { CON } \\
\text { OTRO } \\
\text { FAMILIAR }\end{array}$ \\
\hline Permisivo & 0 & 0 & 0 & 0 \\
\hline Autoritario & 11 & 1 & 1 & 1 \\
\hline Negligente & 5 & 3 & 0 & 0 \\
\hline Democrático & 18 & 10 & 1 & 0 \\
\hline Diferentes & 39 & 0 & 0 & 0 \\
\hline \multicolumn{5}{|c|}{ Nota. La frecuencia refleja a ambos padres. } \\
\hline
\end{tabular}

Por otro lado, en la tabla 2 se muestra la distribución del rendimiento obtenido en comparación con el estilo parental percibido. Se observa que la mayor proporción de quienes obtienen un rendimiento excelente son quienes perciben a ambos padres como democráti$\cos (33.3 \%$ de la muestra), siendo esta diada la que menos alumnos con bajo rendimiento posibilitan, al igual que habiendo un solo padre autoritario. Por otra parte, la acción negligente de uno o ambos padres es la que propicia con más posibilidad la presencia de un bajo rendimiento. Se puede observar que incluso cuando uno de los padres es percibido como democrático, pero el otro como negligente, el bajo rendimiento aún es constante en esta muestra.

A partir de estas observaciones, pareciera que la diada que más posibilita un alto rendimiento, es la compuesta por ambos padres democráticos, seguida por la de padres autoritarios, mientras que las composiciones con menor posibilidad son las que corresponden a estilos negligentes con sus distintas combinaciones, así como cuando hay padres democráticos y autoritarios en el mismo sistema.

Tabla 2.

Frecuencia del estilo parental y rendimiento escolar.

\begin{tabular}{|c|c|c|c|c|c|}
\hline ESTILO PADRE-MADRE & EXCELENTE & Bueno & Elemental & Deficiente & Rendimiento \\
\hline $\begin{array}{l}\text { Permisivo (en } \\
\text { sus distintas } \\
\text { combinaciones) }\end{array}$ & 0 & 0 & 0 & 0 & $N / A$ \\
\hline $\begin{array}{l}\text { Democrático (sólo } \\
\text { uno de los padres) }\end{array}$ & 1 & 5 & 3 & 2 & $\begin{array}{l}\text { Bueno- } \\
\text { excelente }\end{array}$ \\
\hline $\begin{array}{l}\text { Autoritario (sólo } \\
\text { uno de los padres) }\end{array}$ & 1 & 0 & 0 & 1 & Extremos \\
\hline $\begin{array}{l}\text { Negligente (sólo } \\
\text { uno de los padres) }\end{array}$ & 1 & 0 & 0 & 2 & Deficiente \\
\hline $\begin{array}{l}\text { Democrático- } \\
\text { democrático }\end{array}$ & 6 & 4 & 7 & 1 & $\begin{array}{l}\text { Bueno- } \\
\text { excelente }\end{array}$ \\
\hline $\begin{array}{l}\text { Autoritario- } \\
\text { Autoritario }\end{array}$ & 5 & 2 & 2 & 2 & $\begin{array}{c}\text { Bueno- } \\
\text { excelente }\end{array}$ \\
\hline $\begin{array}{l}\text { Negligente- } \\
\text { negligente }\end{array}$ & 0 & 0 & 1 & 4 & $\begin{array}{l}\text { Elemental- } \\
\text { Deficiente }\end{array}$ \\
\hline $\begin{array}{l}\text { Democrático- } \\
\text { autoritario }\end{array}$ & 3 & 7 & 9 & 3 & $\begin{array}{l}\text { Elemental- } \\
\text { Deficiente }\end{array}$ \\
\hline $\begin{array}{l}\text { Democrático- } \\
\text { negligente }\end{array}$ & 0 & 3 & 5 & 4 & $\begin{array}{l}\text { Elemental- } \\
\text { Deficiente }\end{array}$ \\
\hline $\begin{array}{l}\text { Autoritario- } \\
\text { negligente }\end{array}$ & 1 & 1 & 2 & 2 & $\begin{array}{l}\text { Elemental- } \\
\text { Deficiente }\end{array}$ \\
\hline
\end{tabular}

Con el objetivo de comprobar la existencia de diferencias significativas entre los distintos estilos percibidos respecto a su rendimiento académico, se utilizó el estadístico de análisis de varianza de un factor, donde se obtiene que $F(2)=6.879$ y $p=.003$, con lo cual podemos señalar que sí existe una diferencia significativa en el rendimiento académico de los jóvenes a partir de la percepción de los estilos parentales que reciben. Ante este hecho podemos comprobar lo señalado en la hipótesis alterna al referir que existen diferencias significativas cuando los adolescentes perciben el mismo estilo parental y éste es además basado en estilos democráticos.

Con la finalidad de identificar las diferencias significativas entre las diversas relaciones específicas, se aplicó la prueba estadística post hoc de Scheffé, la cual demuestra que la diferencia se encuentra en la comparación del estilo democrático con el negligente, en el estilo autoritario con el negligente y en el negligente con el democrático y autoritario; es decir, el estilo parental negligente es el que resulta el más perjudicial para la variable de rendimiento escolar (tabla 3). 
Tabla 3.

Comparaciones múltiples prueba de scheffé.

\begin{tabular}{|c|c|c|c|c|c|c|}
\hline \multirow{2}{*}{ (I) Estilo } & \multirow{2}{*}{ (J) EstiLo } & \multirow{2}{*}{$\begin{array}{c}\text { DIFERENCIA } \\
\text { DE MEDIAS } \\
(\text { (l-J) }\end{array}$} & \multirow{2}{*}{$\begin{array}{l}\text { ERROR } \\
\text { TíPICO }\end{array}$} & \multirow{2}{*}{ Sig. } & \multicolumn{2}{|c|}{$\begin{array}{l}\text { INTERVALO DE } \\
\text { CONFIANZA A } 95 \%\end{array}$} \\
\hline & & & & & $\begin{array}{l}\text { LÍMITE } \\
\text { INFERIOR }\end{array}$ & $\begin{array}{l}\text { LÍMITE } \\
\text { SUPERIOR }\end{array}$ \\
\hline \multirow{2}{*}{$\begin{array}{l}\text { Permisivo } \\
\text { Autoritario }\end{array}$} & Autoritario & -.076 & .397 & .982 & -1.10 & .95 \\
\hline & Negligente & $1.833^{*}$ & .525 & .006 & .48 & 3.18 \\
\hline \multirow{2}{*}{$\begin{array}{l}\text { Negligente } \\
\text { Democrático }\end{array}$} & Democrático & .076 & .397 & .982 & -.95 & 1.10 \\
\hline & Negligente & $1.909^{*}$ & .560 & .007 & .47 & 3.35 \\
\hline \multirow{2}{*}{ Diferentes } & Democrático & $-1.833^{*}$ & .525 & .006 & -3.18 & -.48 \\
\hline & Autoritario & $-1.909 *$ & .560 & .007 & -3.35 & -.47 \\
\hline
\end{tabular}

*. La diferencia de medias es significativa al nivel .05 .

Con el análisis de homogeneidad fue posible confirmar la diferencia significativa entre el estilo democrático y el autoritario respecto al negligente, corroborando que este último estilo es el más perjudicial (tabla 4).

Tabla 4.

Homogeneidad entre grupos de estilos.

\begin{tabular}{|c|c|c|c|c|}
\hline Estilos & $\begin{array}{l}\text { VIVEN } \\
\text { CON } \\
\text { AMBOS } \\
\text { PADRES }\end{array}$ & $\begin{array}{l}\text { VIVEN } \\
\text { SÓLO CON } \\
\text { MAMÁ }\end{array}$ & $\begin{array}{l}\text { VIVEN } \\
\text { SÓLO } \\
\text { CON } \\
\text { PAPÁ }\end{array}$ & $\begin{array}{c}\text { VIVEN } \\
\text { CON } \\
\text { OTRO } \\
\text { FAMILIAR }\end{array}$ \\
\hline
\end{tabular}

$\begin{array}{lcccc}\text { Permisivo } & 0 & 0 & 0 & 0 \\ \text { Autoritario } & 11 & 1 & 1 & 1 \\ \text { Negligente } & 5 & 3 & 0 & 0 \\ \text { Democrático } & 18 & 10 & 1 & 0 \\ \text { Diferentes } & 39 & 0 & 0 & 0\end{array}$

Al analizar la existencia de alguna diferencia entre el rendimiento escolar de los alumnos que presentan diversidad de estilos parentales tanto en su padre como en su madre, se aplicaron los mismos estadísticos; sin embargo, es posible confirmar que no hay alguna diferencia particular cuando el papel del padres $(F(3)=.956, p=0.421)$ o de la madre $(F(3)=.118, p=0.949)$ es diferente e incide en el rendimiento de los alumnos, de manera que podemos señalar que, habiendo estilos de crianza diversos dentro de un mismo núcleo familiar, los resultados en el rendimiento académico no varía de forma importante.

\section{DISCUSIÓN Y CONCLUSIONES}

A partir de los resultados, es evidente que existe una mayor atención hacia la supervisión escolar de los hijos por parte de la madre (en $65.5 \%$ de los casos lo hacen con frecuencia) con respecto a los padres (61\% lo hacen muy poco); lo cual confirma lo señalado por Chan y Koo (2011) en relación con la existencia de un índice bajo de fracaso escolar cuando los padres mantienen buena supervisión y participación en asuntos escolares de sus hijos; esto se demuestra en este estudio al confirmar dicha relación y déficit cuando sólo alguno de los padres o ninguno de ellos genera dicha supervisión.

Vale señalar que Duchesne y Ratalle (2010) indican de que el estudiante es responsable de esforzarse en su aprendizaje; sin embargo, son los padres quienes deben fomentar responsabilidad, estar al pendiente y animar a sus hijos para que cumplan sus metas educativas y que, como mencionan Tilano, Henao y Restrepo (2009), los padres que se involucran en la supervisión de tareas escolares de sus hijos, combinando los métodos disciplinarios inductivos y refuerzo en lugar de castigo físico, son favorables para el proceso de aprendizaje de los estudiantes y una mayor probabilidad de guiarlos hacia el éxito escolar. Dicha condición es importante tomando en cuenta que, en este estudio, $32.2 \%$ de los alumnos reconoció su mayor dificultad en matemáticas, mientras que $27.8 \%$ en español, siendo ambas materias condiciones básicas para estudios superiores.

El papel de la supervisión por parte de los padres, que a su vez corresponde a un estilo parental vinculado con el desarrollo de los hijos, requiere un hogar bien organizado, que se establezcan las expectativas que pretenden de sus hijos, que pongan reglas claras y que definan con ellos las responsabilidades que les corresponden — donde la más importante es rendir en sus estudios-, y con todo esto promover la autorregulación que es tan esencial para el logro de cualquier propósito en la vida. La supervisión, no sólo escolar sino en la vida cotidiana del hijo, es una práctica parental positiva, la cual comienza a esbozar un camino hacia el estilo democrático (Raya, 2008).

Los datos acerca de los estilos parentales indican que son las madres de familia las que siguen llevando mayor control y atención en la supervisión de asuntos escolares de sus hijos, y los padres son menos participativos; por tanto, se debe atender lo indicado por Díaz y Morales (2011) respecto a la importancia de que ambos padres estén de acuerdo con la forma de educar y que se comprometan a brindar elementos positivos que permitan un mejor desarrollo emocional, social y cognitivo de sus hijos. 
Los hallazgos en esta investigación respecto a los estilos parentales concuerdan con las investigaciones de Tilano, Henao y Restrepo (2009), quienes observaron que los hijos provenientes de un estilo democrático tienen altos porcentajes de buen rendimiento académico, y los que provienen de estilo negligente los peores. Respecto a los estudiantes regidos bajo el estilo autoritario, Torio, Peña y Rodríguez (2008) señalaron que son el segundo lugar en cuanto a rendimiento escolar; sin embargo, en esta investigación dicho estilo tuvo un porcentaje mayor de alumnos con buen y excelente rendimiento $(63.6 \%)$, incluso por encima al de los alumnos regidos con estilo democrático (55.5\%). No obstante, los padres deben tener en cuenta no sólo el resultado académico que se logra con este estilo, sino todas las consecuencias a nivel individual y social, pues la evidencia señala que el estilo autoritario causa un profundo daño psicológico y social al hijo, lo cual va en contra del bienestar global que un padre pretende para sus descendientes.

El punto medular de la investigación fue identificar si los alumnos provenientes de familias con padres de igual estilo parental tienen diferencias significativas en su rendimiento escolar en comparación con los alumnos con padres de diferente estilo parental. En este sentido se encontró que existe una marcada diferencia, pues los padres que comparten estilo autoritario tienen mayormente hijos con buen y excelente rendimiento - de manera similar con el estilo democrático-, y la mayor diferencia está con el estilo negligente; por otra parte, se comprobó que los rendimientos académicos de hijos provenientes de padres con distinto estilo parental no difieren significativamente.

Todo lo anterior indica que si ambos padres ejercen el mismo estilo parental sus hijos tendrán una línea marcada en su desarrollo cognitivo, cada una con sus consecuencias positivas o negativas, pero bien definidas. Esto no sucederá con los alumnos que tienen padres con estilo parental distinto, pues éstos oscilan entre excelente, bueno, regular, mal o deficiente rendimiento debido tanto a dicha diferencia en estilo de los padres como a la interferencia de otras variables.

Con el presente estudio ha sido posible comprobar empíricamente que el rendimiento académico de los adolescentes se ve afectado en función del estilo parental que padecen de sus padres, pero, a diferencia de otros estudios que consideran la percepción de los estilos parentales en conjunto (por diadas padre-madre), aquí ha sido posible realizar el análisis con cada padre como individuo, evidenciando incluso la afectación cuando existen estilos diferentes en esta diada.
Otra cuestión importante en la investigación es que el estilo negligente de los padres ocasiona las consecuencias más negativas en el rendimiento de los hijos, y se deduce que también en otros ámbitos de vida.

Con todos estos hallazgos se puede concluir que el estilo parental es una variable importante para el logro de un buen o mal rendimiento académico; por tanto $-y$ como fue señalado por autores como Hernando, Oliva y Pertegal (2011), Torio, Peña y Rodríguez (2008), Linares (2010) y Raya (2008)—, el estilo democrático es el mejor que los padres pueden ejercer con sus hijos, pues no sólo logra que los chicos tengan buen rendimiento académico, sino que se desarrollen sanamente en todas sus ámbitos.

Lo anterior da pauta para indicar la importancia de tales hallazgos, pues se puede dirigir hacia elementos preventivos y para psicoeducación de los padres, ya que son estos últimos quienes necesitan orientación para guiar a sus hijos; asimismo, los hallazgos pueden dar luz para el desarrollo de nuevas estrategias psicológicas en torno al ámbito familiar, pues - como lo menciona Ruiz (2002) — es importante que los padres tengan una buena comunicación con sus hijos sin importar el tipo de familia que se tenga en la actualidad, aun cuando se viven condiciones tan comunes como el divorcio, el cual no impide que ambas partes se comuniquen y lleguen a acuerdos por el bienestar de sus hijos. El papel óptimo de los padres (sean biológicos o incluso adoptivos) se define hacia prácticas parentales basadas en equidad entre amor y disciplina, considerando que el desafío más grande es que los propios padres busquen orientación para lograr este balance.

Por último, se puede considerar también el elemento a largo plazo, señalado por autores como Enríquez, Segura y Tovar (2013), quienes han indicado que el bajo rendimiento académico augura que el individuo no sea capaz de desarrollarse adecuadamente tanto en su vida personal como en la sociedad, por lo que desbordará en problemas cada vez más graves. En este sentido, describir las condiciones prevalecientes en entornos escolares específicos permite actuar de manera preventiva y ampliar las funciones de la psicología dentro del ámbito educativo para la solución de problemas socialmente relevantes.

Además, se sugiere considerar y promover la inclusión continua de talleres de psicoeducación para expandir la posibilidad de concretar estilos parentales más adecuados al desarrollo de un rendimiento académico óptimo en los adolescentes, tomando en cuenta que la familia asume un papel fundamental en el desarrollo de un buen rendimiento académico, pero que no es el único factor de acción. Es necesario exponer que el resultado de rendimiento académico de los me- 
nores en educación básica y media está sujeto en gran medida a las condiciones burocráticas y administrativas que obligan a las instituciones a seguir marcos de acción encaminados a la "no reprobación" (como indicadores de escuela de calidad), siendo que se hace la promoción de alumnos que, aun sin cubrir los requisitos mínimos de dominio en los temas esenciales, son aprobados en respuesta a tales indicaciones. Ante esta condición, también es posible hacer un seguimiento longitudinal que nos permita conocer el desarrollo del rendimiento académico ante diferentes momentos, con el objetivo de percibir si el impacto del estilo de crianza se ve alterado por cambios en el factor docente, enseñanza y relaciones institucionales que atraviesa el alumno.

\section{Referencias}

Aguilar, J., Valencia, A., y Romero, P. (2007). Impacto de los estilos parentales sobre el ajuste personal, escolar y social entre estudiantes de bachillerato. En J. Aguilar, A. Valencia y Sarmiento C. (Eds.), Relaciones familiares y ajuste personal escolar y social en la adolescencia. Investigaciones entre estudiantes de escuelas públicas (pp. 17-36). México D.F.: Universidad Nacional Autónoma de México, Facultad de Psicología.

Becerra, C. E. (2012), "Variables cognoscitivo-motivacionales relacionadas con el rendimiento escolar de estudiantes de bachillerato", UNAM, FES-Iztacala; disponible en http://www.alfaguia.org/www-alfa/images/ponencias/ clabesIII/LT_1/ponencia_completa_42.pdf.

Bermejo, V. (2011), "Importancia de la familia en el desarrollo socioeducativo del niño", en Revista de la Educación en Extremadura, pp. 1-4; disponible en

http://www.anpebadajoz.es/autodidacta/autodidacta archivos/numero_4_archivos/v_b_minuesa.pdf.

Chan, T. W., y Koo, A. (2011), "Parenting Style and Youth Outcomes in the UK", en European Sociological Review, 27 (3), pp. 385-399; disponible en

http://users.ox.ac.uk/ sfos0006/papers/esr2011.pdf.

Clerici, G., y García, M. J. (2011), Autoconcepto y percepción de pautas de crianza en niños escolares, aproximaciones teóricas, Secretaría de Investigaciones, Facultad de Psicología, UBA, pp. 1-8; disponible en http://www.scielo.org.ar/pdf/anuinv/v17/v17a65.pdf.

Climent, G. I. (2009), “Voces, silencios y gritos: Los significados del embarazo y los estilos parentales educativos", en Revista Argentina de Sociología, 7 (12), pp. 186-213; disponible en http://www.redalyc.org/articulo.oa?id=26912284009.

Consejo Nacional de Fomento Educativo (CONAFE) (2012), Modelo para la participación de padres de familia en la educación inicial y básica del Conafe, pp. 1-65; disponible en http://www.conafe.gob.mx/mportal7/acompaname/ modelo-participacion-padres.pdf.
Covadonga, A. (2001), "Factores familiares vinculados al bajo rendimiento", en Revista Complutense de Educación, 12 (1), pp. 81-113; disponible en

http://revistas.ucm.es/index.php/RCED/article/viewFile/ RCED0101120081A/16850

Cueli, J., y Reidl, L. (1979), Teorías de la personalidad: 3 Alder, Horney (67-76), México, Offset Universal.

Díaz, D., y Morales, M. (2011), “La reciprocidad en la parentalidad y rendimiento académico en adolescentes", en Uaricha Revista 8 (16), pp. 25-35; disponible en http://www.revistauaricha.umich.mx/Articulos/ uaricha 0816 025-035.pdf.

Duchesne, S., y Ratelle, C. (2010), "Parental Vehaviors and Adolescents' Achievement Goals at the Beginning of Middle Scholl: Emotional Problems as Potential Mediators", en Journal of Educational Psichology 2 (102), pp. 497-507; disponible en

http://www.researchgate.net/publication/230703685 Parental_Behaviors and_Adolescents_Achievement Goals_at_the_Beginning_of_Middle_School_Emotional_ Problems_as_Potential_Mediators

Edel, R. (2003), “El rendimiento académico: concepto, investigación y desarrollo", en Revista Electrónica Iberoamericana sobre Calidad, Eficacia y Cambio en la Educación, 1 (2), pp. 1-15; disponible en

http://repositorio.uam.es/bitstream / handle/10486/660693/REICE 12 7.pdf? sequence=1.

Enríquez, D. J., y Robles, S. (2013), “El papel de la cercanía entre hermanos sobre la conducta sexual protegida", en Acta de Investigación Psicológica, 3 (2), pp. 1092-1107, México, UNAM; disponible en

http://www.scielo.org.mx/pdf/aip/v3n2/v3n2a4.pdf

Enríquez, C. L., Segura, A. M., y Tovar, J. R. (2013), Factores de riesgo asociados a bajo rendimiento académico en escolares de Bogotá, Investigaciones Andina 15 (26), pp. 653-666; disponible en

http://www.redalyc.org/articulo.oa?id=239026287004

Fernández, I. (2011), “La importancia de la familia en la educación infantil", pp. 1-55; disponible en

http://www.eduinnova.es/monografias2011/mar2011/ familia.pdf

González, J. A. (2008), “El rendimiento escolar: un análisis de las variables que lo condicionan", en Revista GallegoPortuguesa de Psicología de Educación, 7 (8), pp. 247-258; disponible en

http://ruc.udc.es/bitstream/2183/6952/1/RGP_9-17.pdf

Guallpa, N. C., y Loja, B. L. (2015), “Estilos de crianza de los padres de estudiantes con bajo rendimiento", tesis de licenciatura en Psicología, Universidad de Cuenca; disponible en

http://dspace.ucuenca.edu.ec/jspui/ bitstream/123456789/21872/1/TESIS.pdf

Hernández, R., Fernández, C., y Baptista, P. (1991), Metodología de la investigación, México, McGraw Hill.

Hernando, A., Oliva, A., y Pertegal, M. A. (2011), "Variables familiares y rendimiento académico en la adolescencia", en Estudios de Psicología, 33 (1), pp. 51-65; disponible en http://www.uhu.es/angel.hernando/documentos/2012 Estudios de Psicologia.pdf 
Instituto Nacional para la Evaluación de la Educación (INEE) (2003), “¿Qué hace a una escuela, una buena escuela? Calidad educativa, su evaluación y sus principales retos para México; disponible en

http://www.inee.edu.mx/images/stories/Publicaciones/ Textos divulgacion/Este Pais/buena escuela/buena escuela.pdf

Ishak, Z., Low, S. F., y Lau, P. L. (2011), "Parenting Style as a Moderator for Students' Academic Achievement", en Journal of Science Education and Technology, pp. 487493; disponible en

http://www.researchgate.net/profile/Zahari Ishak/ publication/225144393 Parenting Style as a Moderator_for_Students_Academic_Achievement/ links/54cae6830cf2c70ce52444fa.pdf

Kleisner, A., y MacPhee, D. (2011), How Home gets to School: Parental Control Strategies Predict Children's School Readiness, Colorado State University, pp. 355-364; disponible en

http://www.researchgate.net/publication/251599998 How_home_gets_to_school_Parental_control_strategies predict children's school readiness

Linares, E. M. (2010), Influencia del Estilo Educativo Familiar en el Rendimiento Académico del Alumnado, Universidad de Almería, pp. 1-26; disponible en

http://repositorio.ual.es:8080/jspui/ bitstream/10835/2416/1/Trabajo.pdf

Madrigales, C. N. (2012), "Autoconcepto en adolescentes de 14 a 18 años, tesis de licenciatura en Psicología, Universidad Rafael Landivar; disponible en

http://biblio3.url.edu.gt/Tesis/2012/05/42/MadrigalesCeily.pdf

Pelayo, J. M. (2013), “Motivación para el cambio”, Universidad de Oviedo, pp. 1-16; disponible en

http://www.unioviedo.es/psiquiatria/docencia/material/ PSI\&COMUNICACION/2013-14/6PC-MOTIVACION.pdf

PISA (2011), “¿Qué pueden hacer los padres para ayudar a sus hijos a tener éxito en los centros educativos?, pp. 1-4; disponible en

http://www.oecd.org/pisa/pisaproducts/ pisainfocus/49460778.pdf

Ramírez, M. A. (2005), “Padres y desarrollo de los hijos: prácticas de crianza", en Estudios Pedagógicos 2 (16), pp. 167-177; disponible en

http://www.redalyc.org/articulo.oa?id=173519073011

Raya, A. F. (2008), "Estudio sobre los estilos educativos parentales y su relación con los trastornos de conducta en la infancia", tesis doctoral, Universidad de Córdoba.

Rezk, M. (2015), “Auto-control: nociones básicas e investigación fundamental", en Revista Latinoamericana de Psicología, 8 (3), pp. 389-397; disponible en

http://www.redalyc.org/pdf/805/80580303.pdf
Rivadeneira, J. (2013), "El funcionamiento familiar, los estilos parentales y el estímulo al desarrollo de la teoría de la mente: efectos en las criaturas sordas y oyentes", tesis doctoral, Universidad Autónoma de Barcelona; disponible en

http://www.tdx.cat/bitstream/handle/10803/117201/ jrv1de1.pdf?sequence=1

Rodríguez, C., y Caño, A. (2012), "Autoestima en la adolescencia: análisis y estrategias de intervención", en International Journal of Psychology and Psychological Therapy, 12 (3), pp. 389-403; disponible en

http://www.ijpsy.com/volumen 12/num3/337/ autoestima-en-la-adolescencia-anlisis-ES.pdf

Roksa,J.,yPotter,D.(2011),“ParentingandAcademicAchievement: Intergenerational Transmission of Educational Advantage", en American SociologicalAssociation 84 (4), pp. 299-321; disponible en

h t t p://soe.sage pub.com/content / early/2011/08/16/0038040711417013

Ruiz, M. E. (2002), "Factores que influyen en el rendimiento escolar de los adolescentes", tesis de licenciatura en Psicología, Universidad Pedagógica Nacional; disponible en http://200.23.113.59/pdf/19186.pdf

Ruiz, P. J. (2013), Los Problemas Escolares en la Adolescencia, Servicio Madrileño de Salud, 8 (2), pp. 117-127; disponible en

http:/www.codajic.org/sites/www.codajic.org/files/ Los $\% 20$ escolares $\% 20 \%$ en $\% 201$ a $\% 20$ Adolescencia $\% 20$ PJ\%20ruiz\%20Lazaro.pdf

Secretaría de Educación Pública (SEP) (2012), Boletín Informativo Estadístico, pp. 1-10; disponible en

http://www.sepyc.gob.mx/documentacion/boletinnum2.pdf

Tilano, L. M., Henao, G. C., y Restrepo, J. (2009), Prácticas educativas familiares y desempeño académico en adolescentes escolarizados en el grado noveno de instituciones educativas oficiales del municipio de envigado, Universidad de San Buenaventura, 9 (1), pp. 3551; disponible en

http://web.usbmed.edu.co/usbmed/elagora/htm/ v9nro1/pdf/cap2.pdf

Torio, S., Peña, J. V, y Rodríguez, M. C. (2008), Estilos educativos parentales, revisión bibliográfica y reformulación teórica, Universidad de Oviedo, pp. 1-28; disponible en http://dialnet.unirioja.es/servlet/articulo?codigo=2718367

Valdés, A. A., Carlos, E. A., y Ochoa, J. M. (2010), “Características emocionales y conductuales de hijos de padres casados y divorciados", en Revista Internacional de Psicología y Educación, 1 (12), pp. 117-134; disponible en http://www.redalyc.org/articulo.oa?id=80212393007 


\section{Dimensión Cuantitativa}

\section{Perfil de Evaluación entre pares}
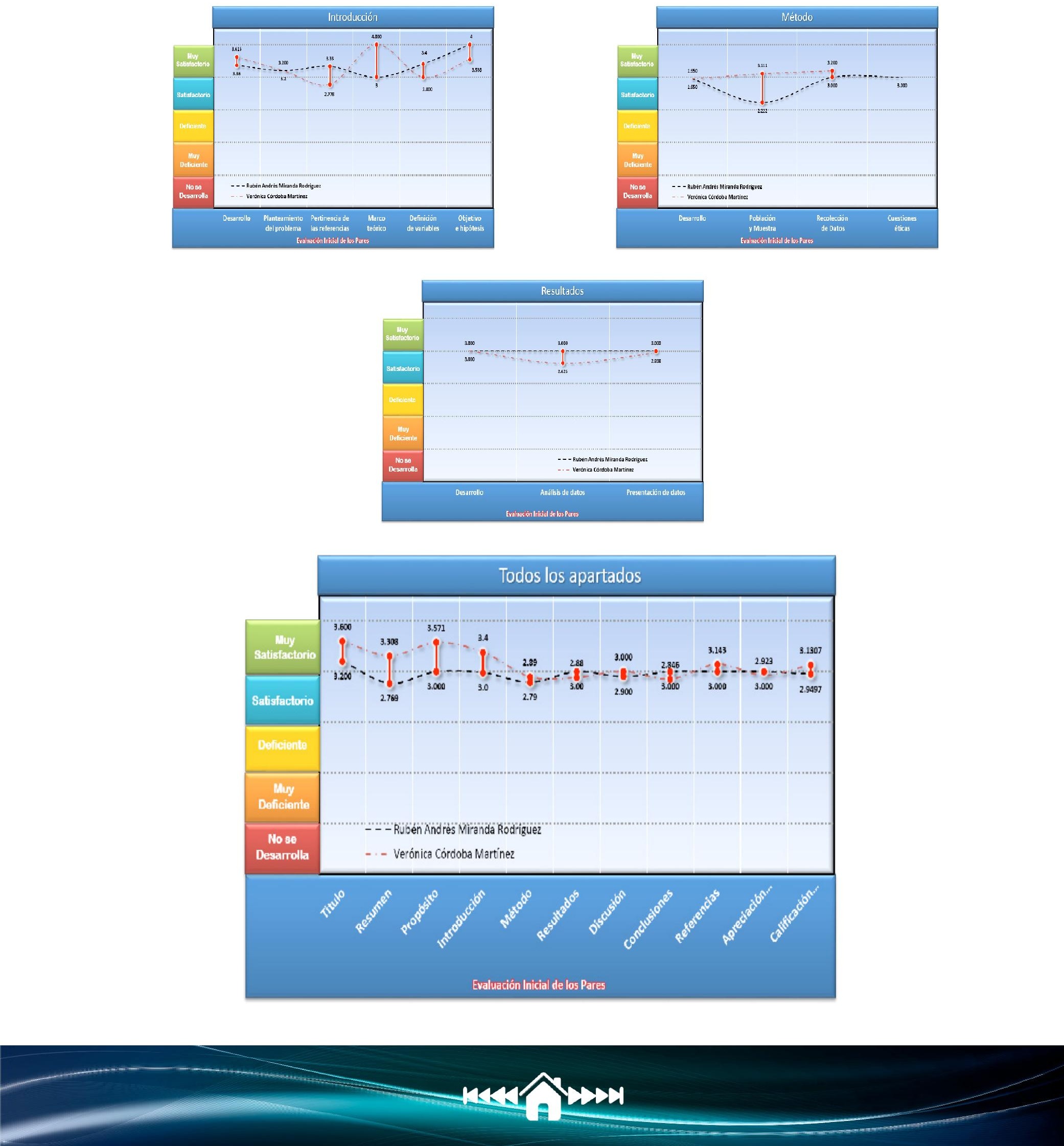


\section{Índice de Concordancia}

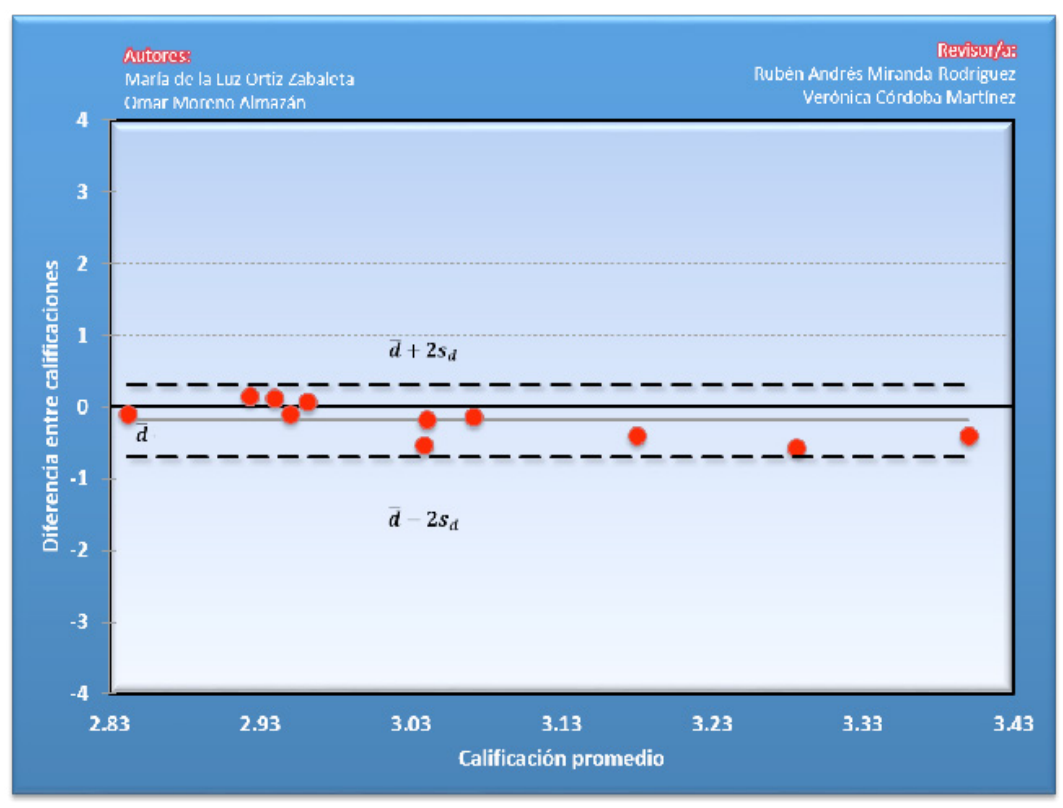

Índice de Acuerdo
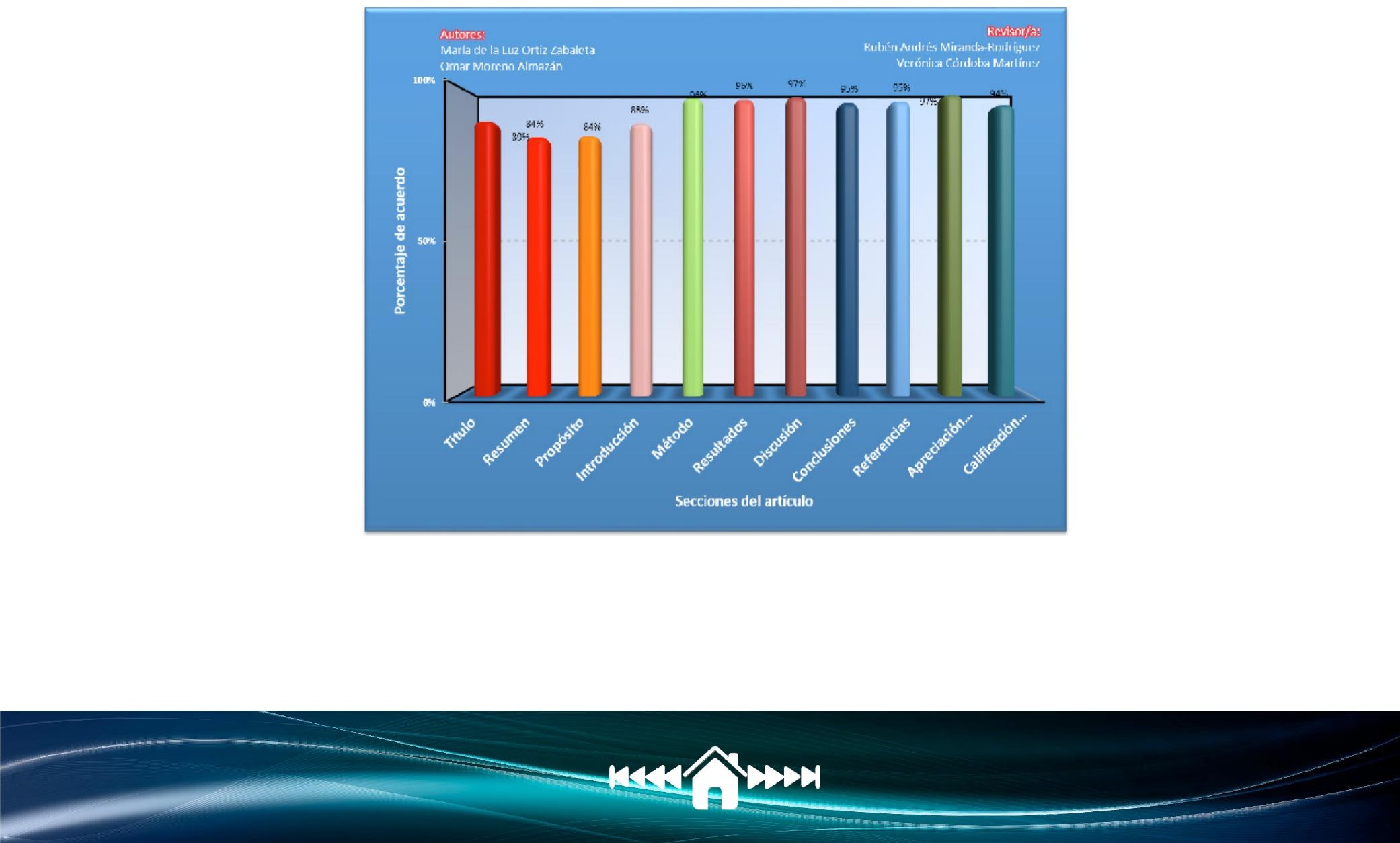
Dimensión Cualitativa

\begin{tabular}{|c|c|}
\hline Revisor 1 & Revisor 2 \\
\hline Rubén Andrés Miranda-Rodríguez & Verónica Córdova \\
\hline \multicolumn{2}{|c|}{ Título/Autoría } \\
\hline Sin comentario. & $\begin{array}{l}\text { El título refleja las variables analizadas en la investiga- } \\
\text { ción. }\end{array}$ \\
\hline \multicolumn{2}{|c|}{ Resumen } \\
\hline $\begin{array}{l}\text { Cambiar las palabras clave por unas que no estén en el } \\
\text { título de la investigación. Mencionar en el resumen que } \\
\text { se usó un ANOVA para el análisis de las diferencias. }\end{array}$ & $\begin{array}{l}\text { Menciona objetivo, muestra, instrumento, resultados y } \\
\text { conclusiones sin embargo excede las } 150 \text { palabras per- } \\
\text { mitidas en el resumen con } 77 \text { palabras. }\end{array}$ \\
\hline \multicolumn{2}{|c|}{ Próposito del Estudio } \\
\hline $\begin{array}{l}\text { Se usan muchas palabras en primera persona, recordar } \\
\text { que tiene que ser en tercera persona o impersonal. }\end{array}$ & $\begin{array}{l}\text { El artículo puede ser un aporte dentro del ámbito edu- } \\
\text { cativo, cumple con su propósito de estudio. Sin mayo- } \\
\text { res observaciones }\end{array}$ \\
\hline \multicolumn{2}{|c|}{ Introducción } \\
\hline $\begin{array}{l}\text { Se sugiere detallar conceptualmente cada variable y su } \\
\text { posible influencia de otros factores. }\end{array}$ & $\begin{array}{l}\text { Se recomienda usar referencias más actuales } 2014,1215 \\
\text { y } 2016 \text {. Se recomienda hacer mayor uso de la redacción } \\
\text { de los autores a lo largo de la introducción (evitando el } \\
\text { párrafo textual y la cita) }\end{array}$ \\
\hline \multicolumn{2}{|c|}{ Método } \\
\hline $\begin{array}{l}\text { Se sugiere tener cuidado con la especificación del tipo } \\
\text { de muestreo, ya que si es probabilístico aleatorio sim- } \\
\text { ple, se tienen que especificar los cálculos pertinentes } \\
\text { para la elección de la muestra (n) y cómo se tuvo el } \\
\text { control de la población }(\mathrm{N}) \text {. En caso de no ser así, se } \\
\text { trata de un muestreo no probabilístico intencional, con } \\
\text { sus respectivos criterios de inclusión. Se considera que } \\
\text { las hipótesis se tienen que redactar en presente, impli- } \\
\text { cando contundencia. }\end{array}$ & $\begin{array}{l}\text { Se recomienda fundamentar claramente los procedi- } \\
\text { mientos para la selección y/o se justificar el tamaño de } \\
\text { la muestra. Describir claramente los procedimientos } \\
\text { para la protección y la forma de obtención del consen- } \\
\text { timiento informado. }\end{array}$ \\
\hline \multicolumn{2}{|c|}{ Resultados } \\
\hline $\begin{array}{l}\text { Las especificaciones en las tablas son claras, sin embar- } \\
\text { go no se encuentran en formato APA. Es necesario mo- } \\
\text { dificarlas a este estilo. }\end{array}$ & $\begin{array}{l}\text { La sección de resultados se presenta de manera clara y } \\
\text { concisa }\end{array}$ \\
\hline
\end{tabular}




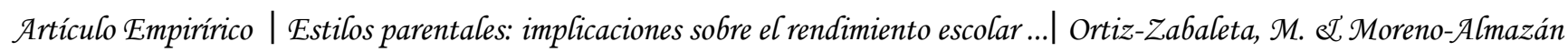

\begin{tabular}{|c|c|}
\hline Revisor 1 & Revisor 2 \\
\hline \multicolumn{2}{|c|}{ Discusión } \\
\hline $\begin{array}{l}\text { En cada investigación, es importante especificar limi- } \\
\text { tantes, para convertirlas en sugerencias de estudios } \\
\text { posteriores. }\end{array}$ & $\begin{array}{l}\text { Se discuten primero los resultados de la investigación } \\
\text { y se toman otros referentes para apoyar o contrastar } \\
\text { los mismos, se recomienda usar estudios más actuales } \\
(2015,2016) \text {. }\end{array}$ \\
\hline \multicolumn{2}{|c|}{ Conclusiones } \\
\hline Son muy buenas conclusiones. & $\begin{array}{l}\text { Se menciona hasta el último párrafo las contribucio- } \\
\text { nes, en eje de investigación para la psicología, se podría } \\
\text { profundizar en este punto. No se mencionan deficien- } \\
\text { cias del estudio ni como podrían resolverse a futuro. }\end{array}$ \\
\hline \multicolumn{2}{|c|}{ Referencias } \\
\hline $\begin{array}{l}\text { Habría que revisar la cuestión del espacio entre renglo- } \\
\text { nes, únicamente. }\end{array}$ & Sin mayores comentarios \\
\hline
\end{tabular}

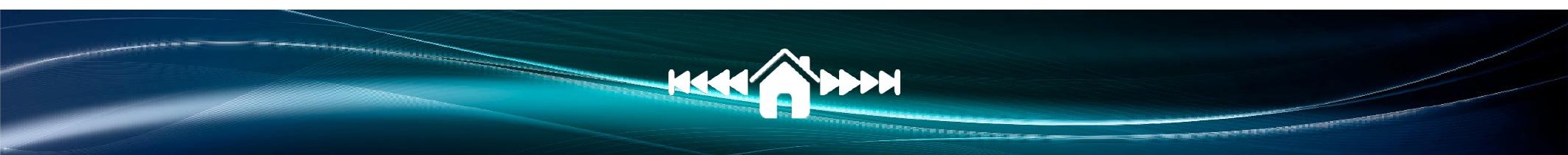


Revista Digital Internacional de Psicología y Ciencia Social |Vol. 2 | Núm. $1 \mid$ Enero-Junio 2016 | ISSSN 1405-5802

Historia del Proceso

\section{EDITORIAL}

